American Journal of Applied Sciences 8 (11): 1228-1231, 2011

ISSN 1546-9239

(C) 2011 Science Publications

\title{
Zero Geometry, Zero Space Time, The Seeds of The Final Theory
}

\author{
Kambiz-Afrasiabi \\ Department of Medicine, \\ University of California, Irvine, California, USA
}

\begin{abstract}
Problem statement: Lack of understanding And scientific formulation of zero geometry and zero space-time and the truth about initiation conditions would continue to keep us all in dark and we would continue to create more theories defining our observations about the known universe which could Indeed be part of our illusionary perception of reality And as long as they continue to align themselves with our observations we will continue to obey and propagate them. Approach: Second law of thermodynamics which was considered to be the most fundamental law governing The known universe and its behavior and at all levels from micro to macro cosmos was itself subject to such flaws simply because it was nonexistent in zero space-time and it reaches infinity at leap out of zero state bordered by infinity wall (the outermost border of the leap universe) in no time. This law dictates that in the known universe/post big bang universe which was around 13.4 billion light years old the index of instability which inversely correlates with free energy incessantly increases following its inception, though we were in dark regarding the initiation condition of the known universe and relation of initiation condition with entropy and This needs to be understood, dissected and formulated Into the new physics on an urgent basis. Results: Big bang which had been proposed to give rise to the known universe presumably was the product of collision of two p-branes and this collision was expected to be similar in nature to the collision of biological p-branes In which case one p-brane impregnates the other and I have alluded to that in my previous publications; Following collision of cosmic p-branes the free energy of impregnated p-brane increases from zero to infinity in no time and because the cosmic p-branes were the residents of zero geometry and zero space-time in which all the values are zero, i.e., free energy and entropy were paradoxically both zero and because p-brane collision leads to disentanglement and decohe scence of these two values, leap out of zero geometry and space-time happens to re entangle these two values as a result of which leap universe was born the outermost border of which was infinity wall with infinity as the value of entropy however this creates another paradox simply because at that point the free energy was zero and entropy is infinity and again these two universal values find themselves disentangled and decohesced and this time the massive force of repulsion at infinity wall which was what I consider dark energy and was the source of massive negative gravity would create the second phase of the non-zero universe, i.e., post leap universe which one more time was aiming at entanglement, this time infinite entropy shifting to zero value to regain its original value of zero space-time universe, this would take the post leap Universe to zero geometry border but this move would Increase the free energy to infinity and that creates big Bang universe/phase 3 universe the goal of which was entanglement of free energy with zero entropy and this Ping pong reaction between the zero geometry partners in search of entanglement and cohescence in non-zero universe would continue indefinitely and this time expansion of the known universe through corridors of the dark matter creates post big bang universe as a result of which the four major forces of the known universe/regular matter would be born, i.e., would get separated into four different entities and the post big Bang universe would pursue its goal of decreasing the free energy which would reach zero (its original zero Geometry value) at the fire wall/repulsion trigger zone of the infinity wall and another round of repulsion, crunch, big bang and expansion would ensue; the engine of the above sequence of events following collision of the cosmic p-branes was massive increase in free energy in contradistinction to the biouniverse in which fertilization/collision of biological p-branes leads to a massive and sudden decrease in free energy of the fertilized ovum/the biological p-brane,
\end{abstract}


Am. J. Applied Sci., 8 (11): 1228-1231, 2011

(Afrasiabi, 2010). And thus triggering the big bang of recurrent rounds of mitosis the objective of which was to increase the free energy/decrease the entropy of the future generation cells simply because the living universe was an anti entropy machinery by nature and the known universe was an entropy promoting one. Conclusion/Recommendations: In living universe any event that inadvertently increases the entropy compared with the expected and normal entropy for that stage in life cycle can lead to a catastrophic disease such as cancer and auto- immune disorders if the corrective Mechanisms can not capture or correct the error and in the known/ non-living (post big bang) universe which follows the post leap universe any event that opposes the incessant increase in entropy/decrease in free energy can lead to cataclysmic events such as contraction of space-time/formation of baby black holes or massive and Aberrant outburst of high energy particles. This anti parallelism of the mechanism of formation and evolution of events in the living and non living universes and the opposite role that the second law of thermodynamics is playing in their births and evolution would offer us an exceptional opportunity to find solutions to many of the puzzles and unknowns regarding their path in the past and future and finding solutions to their aberrancies from cure of cancer as $\mathrm{i}$ alluded to in my previous publication all the way to time travel and migration out of milky galaxy .

Key words: Black hole, p-brane, zero geometry, zero space-time, planck wall, dark energy, dark matter, big bang, negative gravity, quantum universe, parallel universes

\section{INTRODUCTION}

Relativity breaks down at initiation and a theory that cannot Explain the initiation condition can not be a complete theory And its validity becomes doubtful and indeed dark matter, dark energy and black holes were not Predicted by this theory even if we consider it valid for the Explanation of the behavior of around $4 \%$ of the known universe/regular matter; quantum mechanics which is based on uncertainty, entanglement and decohescence has had historical difficulty grasping the missing links with guts/grand unified theory, there is an urgent need for formulation of a new physics which could build new frontiers and break The old barriers and rules. Dissection and formulating of zero geometry and zero space-time physics would create the Foundation of the final theory and would offer us such an exceptional opportunity; definitions of dark energy, dark matter and the four major known forces of the known universe (around $4 \%$ of the universe), namely electromagnetic force, gravity, weak and strong nuclear forces which are different versions and conversions and Ramifications of the same original leap energy in the context of the seeds of the final theory and the conclusion about Initiation and end conditions would become possible with this kind of understanding; based upon the above mentioned principles birth of the known universe is triggered by a sudden and inadvertent increase in free energy of the impregnated p-brane and the ensuing leap out of zero geometry. By the token of this theory p-branes are the residents of zero geometry and zero space time in which all values are zero and the purpose of big bang generated universe/the third (phase) universe would be unlike that of the big bang of mitosis following fertilization/collision of biological pbrines, i.e., rise in entropy of the universe that it creates following the leap and post leap states, this would give the second law of thermodynamics validity even before big bang in contradistinction to the current thinking. So at the post leap state the entropy of the universe would continue to decrease compared with the leap state itself which is bordered by infinity wall, though it is increasing compared with zero geometry in the post big bang era following original repulsion generated by massive anti- gravity of the infinity wall and the ensuing expansion preceded by big crunch and big bang and this Is one of the hallmarks of the quantum universe in which the original disentanglement of zero free energy from zero Entropy happened following collision of the zero geometry residents, namely the p-branes. The theory of big bang and Infinite number of other big banks and their relationship with P-brane collisions has all become the hottest issue in science and the latest theory in the explanation of the initiation condition though the true understanding of the conditions of Initiation has been missing until now (Hawking, 1988; Miller, 1995; Weinberg, 1977;1992) this kind of understanding would lead to a fundamental and revolutionary change in our understanding of the known universe as well as other universes including the universe of living things and their aberrancies including catastrophic disorders such as cancer, (Afrasiabi, 2011), all the way to the possibility of time travel and breaking the walls of photon prison and clearly lack of this kind 
of understanding has led to generation of many false theories in different realms of science from micro to macrocosms level. One might start to think that the leap out of zero geometry of nothingness ending in big bang instantly took the system into infinity which is defined as any non-zero state both as far as entropy and birth of space time are concerned and the born universe extended to its outer most borders/infinity wall in no time.

Thus the increase in entropy of the quantum universe applies To that of the born universe/the leap universe, the one that Has reached infinity wall in no time following collision of $\mathrm{p}$-branes Compared with that of zero geometry and not that of The post leap universe compared with leap universe, though Post leap universe entropy is higher compared with zero geometry following original repulsion by negative gravity of Infinity wall and re expansion preceded by big crunch and big bang until it hits the repulsion zone of the infinity wall again which normally leads to crunch and another cycle of expansion all fueled by original disentanglement in zero Space-time and this misunderstanding of sequence of events could lead to huge flaws in our understanding of the universe and create a major handicap in formulating the right type of physics, a physics that would enable us to pass through the walls of space-time and create the foundations of grand unified theory. I would like to touch on a few major topics based on this universal blueprint including Planck wall, super string theory, dark energy, dark matter, relation of black holes with space-time, super symmetry, super gravity, inflationary universe and bubble theory; starting with Planck wall which has been defined as 10-43 sec following big bang; we have learned that the events before that time are not comprehendible, though science of physics can understand the backward sequence of events until that moment, though Indeed this understanding is in need of understanding of the Infinity wall of the leap universe beyond which it is impossible to penetrate and away from which is a swift bounce back towards zero geometry as a result of the massive repulsive force of the infinity wall; that massive repulsive force is what I consider a fraction of dark energy from which dark matter is derived during repulsion by de $=$ dmxdfrk, in this equation dark matter is equal to dark energy divided by dark force of repulsion multiplied by a constant derived from the disentanglement force. Expansion resumes through the corridors of the dark matter back again towards the infinity wall which is what $\mathrm{i}$ consider the outermost border of dark energy zone at an accelerating pace generated by original repulsive force/negative gravity leading to successions of crunch and accelerating expansion. So indeed pbrane collisions could be the source of the melody of parallel universes following release of massive amount of energy leading to leap, repulsion, crunch, bang, expansion, repulsion, crunch as their different notes; through the above example we could start to understand the true meaning of dark energy, dark matter, inflationary universe/the process of formation of leap universe, bubble, (gut-A), which could be phase one universe (leap universe, super string which could be strings of vibrations originating from negative gravity of the infinity wall on which regular matter passing through dark matter corridors gets shaped, super gravity which could be the negative gravity originating from The infinity wall, super symmetry which could reflect the symmetrical leap and post leap universes, black holes in which photonic time comes to an end but still are like ships in the ocean of space-time; and accelerated pace of expansion of the Post big bang universe which is the result of decreasing force of attraction of galaxies as they get farther from one Another and not the attractive force of dark energy, indeed dark energy is repulsive only. Now we can see that lack of understanding of the truth about initiation and end conditions has led to a lot of false definitions which are being taught in our universities every day.

\section{MATERIALS AND METHODS}

Generation of initiation or near initiation condition, i.e., zero or near zero space-time would lead to or promote re entanglement and cohescence of the two most fundamental universal values, namely free energy and entropy which have been in search of zero for ages following the original leap into non-zero universe .

\section{RESULTS}

This would lead to shrinkage/shift of the surrounding universe to near zero and opening of the gate of near zero dimensions/worm holes and would make migration to the other side of the universe feasible.

\section{DISCUSSION}

We are equipped with both the imagination, desire to achieve our far reaching goals and a lot of scientific advances; The time is ripe For proceeding with mathematical formulation of the new physics by 
close collaboration of mathematicians and physicist globally based on the above mentioned principles of the three phase universe.

\section{CONCLUSION}

Understanding, dissection and mimicking zero geometry and zero space-time condition and its scientific formulation would prepare the foundations of the new physics and the seeds of the grand unified theory which is urgently needed. Human race is at a critical cross road and migration outside of this corner of universe could lead to preservation of the highest gift of evolution, namely life. Current physics plagued by lack of understanding of the initiation condition has taken us into wrong and dark paths characterized by misunderstandings and false definitions. re entanglement of the two most fundamental elements of the universe, i.e., free energy and entropy which became disentangled by the collision of p-branes and incarceration in space-time could potentially get reversed if we could generate zero or near zero spacetime condition and by doing so we could open a new chapter in the evolution of mankind, a chapter which could lead to the creation of grand unified theory, migration to other corners of universe, understanding and cure of catastrophic diseases such as cancer and propagation of the most sacred gift of evolution to safer corners of universe and most importantly perception of the truth prevailing the universe .

\section{REFERENCES}

Afrasiabi, K., 2010. Quantum stem cell in the infinity loop: A new concept for our understanding of the normal state and cancer. Online J. Biol. Sci., 10: 60-65. DOI: $10.3844 /$ ojbsci.2010.60.65

Afrasiabi K., 2011. Entropyomics as the blueprint of the logic of normal cell division and malignancy. Online J. Biol. Sci., 11: 23-26. DOI: 10.3844/ojbsci.2011.23.26

Hawking, S.W., 1988. A Brief History of Time. 10th Edn., Bantam Books, New York, ISBN-10: 0553380168, pp: 212.

Miller, C., 1995. Cosmic Hide and Seek: The Search for the Missing Mass. Chris Miller.

Weinberg, S., 1977. The First Three Minutes: A Modern View of the Origin of the Universe. 1st Edn., Basic Books, New York, ISBN-10: 0465024351, pp: 188.

Weinberg, S., 1992. Dreams of a Final Theory. 1st Edn., Pantheon Books, New York, ISBN-10: 0679419233, pp: 334. 